\title{
Desempenho de escolares bons leitores, com dislexia e com transtorno do déficit de atenção e hiperatividade em nomeação automática rápida
}

\section{Performance of good readers, students with dyslexia and attention deficit hyperactivity disorder in rapid automatized naming}

\author{
Simone Aparecida Capellini ${ }^{1}$, Tais de Lima Ferreira ${ }^{2}$, Cíntia Alves Salgado ${ }^{3}$, Sylvia Maria Ciasca ${ }^{4}$
}

\begin{abstract}
RESUMO
Objetivos: Caracterizar o desempenho de escolares com transtorno do déficit de atenção e hiperatividade e com dislexia em nomeação automática rápida e comparar o desempenho destes escolares com aqueles que lêem conforme o esperado para idade e escolaridade. Métodos: Participaram deste estudo 30 escolares na faixa etária de oito a 12 anos de idade de $2^{\mathrm{a}}$ a $4^{\mathrm{a}}$ séries do ensino público fundamental, divididos em três grupos de 10 escolares, sendo um grupo de escolares com transtorno do déficit de atenção e hiperatividade, um grupo de escolares com dislexia e um grupo de escolares com bom desempenho escolar. Resultados: Os resultados revelaram diferenças estatisticamente significantes, evidenciando desempenho superior do grupo controle em relação ao grupo de escolares com transtorno do déficit de atenção e hiperatividade e ao grupo de escolares com dislexia nos subtestes de cores, dígitos, letras e objetos; e desempenho superior dos escolares do grupo com transtorno do déficit de atenção em relação ao grupo de escolares com dislexia em nomeação automática rápida. Conclusão: O presente estudo demonstrou que os escolares que lêem conforme o esperado para idade e escolaridade apresentaram melhor desempenho em relação ao grupo de escolares com transtorno do déficit de atenção e hiperatividade e o grupo com dislexia, demonstrando que tal habilidade pode ser considerada um pré-requisito para o desempenho em leitura.
\end{abstract}

DESCRITORES: Transtorno da falta de atenção com hiperatividade; Dislexia; Baixo rendimento escolar; Leitura

\section{INTRODUÇÃO}

O Transtorno de Déficit de Atenção e Hiperatividade (TDAH) é o distúrbio neuropsiquiátrico mais comum da infância e está incluído entre as doenças crônicas mais prevalentes entre escolares. Em amostras não referidas, esti-

(1) Pós-Doutoranda em Ciências Médicas da Faculdade de Ciências Médicas da Universidade Estadual de Campinas - UNICAMP - Campinas (SP), Brasil; Docente do Departamento de Fonoaudiologia da Faculdade de Filosofia e Ciências da Universidade Estadual Paulista - UNESP - Marília (SP), Brasil. (2) Aluna especial do Programa de Pós-Graduação em Ciências Médicas da Faculdade de Ciências Médicas da Universidade Estadual de Campinas UNICAMP - Campinas (SP), Brasil

(3) Pós-graduanda do Programa de Pós-Graduação em Ciências Médicas da Faculdade de Ciências Médicas da Universidade Estadual de Campinas UNICAMP - Campinas (SP), Brasil.

(4) Livre Docente em Ciências Médicas da Faculdade de Ciências Médicas da Universidade Estadual de Campinas - UNICAMP - Campinas (SP), Brasil. Trabalho realizado no Laboratório de Pesquisa em Distúrbios, Dificuldades de Aprendizagem e Transtornos da Atenção do Departamento de Neurologia da Faculdade de Ciências Médicas da Universidade Estadual de Campinas UNICAMP - Campinas (SP), Brasil.

Endereço para correspondência: Simone Aparecida Capellini. R. Bartolomeu de Gusmão, 10-84, Jardim América, Bauru - SP, CEP 17017-

326. E-mail: sacap@uol.com.br

Recebido em: 24/10/2006; Aceito em: 25/5/2007 ma-se que $3 \%$ a $6 \%$ das crianças em idade escolar apresentam TDAH ${ }^{(1)}$.

A dislexia é caracterizada como transtorno da leitura e da escrita, que interfere no rendimento escolar, deixando-o inferior ao esperado em relação à idade cronológica do indivíduo, ao seu potencial intelectual e à sua escolaridade ${ }^{(2)}$. Estima-se que afete em torno de 5 a $10 \%$ de escolares ${ }^{(3-5)}$.

Tanto o TDAH como a dislexia são condições genéticoneurológicas que podem apresentar, em sua história acadêmica, o fracasso escolar, quer seja determinado por alterações na entrada, como ocorre no TDAH ou no processamento cognitivo da leitura, como na dislexia.

Nos últimos 30 anos, as pesquisas têm enfocado a relação entre os processamentos perceptuais, lingüísticos e cognitivos envolvidos na leitura. Como consequiência destas pesquisas, hoje é possível a aplicação de procedimentos de avaliação que possibilitam aos pesquisadores verificar o uso de habilidades do processamento fonológico, como a consciência fonológica, a memória de trabalho e a nomeação rápida em populações de leitores proficientes ou com alterações no processo de aprendizagem na leitura. Uma das habilidades do processamento fonológico mais estudadas internacionalmente no período citado foi a nomeação rápida.

Os estudos envolvendo a nomeação rápida tiveram seu 
início em 1974. O primeiro teste descrito na literatura ${ }^{(6)}$ foi elaborado a partir de estudos anteriores sobre a dificuldade de nomeação de indivíduos com dislexia em tarefas de nomeação automática, os quais verificaram que o desempenho observado era significativamente inferior quando comparado aos dos sujeitos sem o quadro de dislexia.

Entretanto, a literatura especializada demonstra que os estudos sobre a nomeação rápida divergem quanto à sua vinculação ao processamento fonológico ou a velocidade no processamento da informação ${ }^{(7)}$. Para alguns autores ${ }^{(8-11)}$, a nomeação rápida é uma habilidade do processamento fonológico, enquanto que para Wolf e Bowers ${ }^{(12)}$ a mesma faz parte da velocidade do processamento da informação. Apesar desta divergência, os pesquisadores envolvidos nestas duas vertentes são unânimes em afirmar que a nomeação rápida possui relação com a leitura, principalmente em habilidades de decodificação, fluência e compreensão.

No que se refere ao processamento fonológico, o mesmo faz parte do processamento auditivo ligado ao domínio da leitura e escrita ${ }^{(13)}$. Para os autores, o processamento fonológico refere-se à informação fonológica recebida auditivamente, que está diretamente relacionada ao desenvolvimento da linguagem oral e da linguagem escrita em um sistema de escrita alfabética. A consciência fonológica, a memória fonológica e a nomeação rápida compõem o processamento fonológico.

De acordo com os pressupostos teóricos desenvolvidos pelos autores citados anteriormente, a nomeação rápida é entendida como parte da velocidade de processamento da informação. Baseado na hipótese do duplo-déficit para a dislexia do desenvolvimento, na qual os déficits fonológicos e os processos subjacentes aos déficits de nomeação são descritos como duas fontes independentes dos distúrbios da leitura, existem três subtipos de déficits: déficits apenas em nomeação rápida; apenas na consciência fonológica; nas duas habilidades cognitivas, todos com conseqüentes alterações na leitura ${ }^{(14)}$

Ainda segundo os autores ${ }^{(12)}$, as habilidades necessárias para a nomeação rápida envolvem: atenção ao estímulo, processos visuais responsáveis pela discriminação, identificação da letra e o seu padrão, integração de características visuais e informação de padrão visual com estocagem de representações ortográficas, integração de informação visual com representações fonológicas estocadas, acesso e recuperação fonológica, ativação e integração da informação semântica e conceitual e ativação motora para a articulação.

A nomeação rápida também é descrita na literatura como "nomeação rápida automática"(6), "recodificação fonológica de acesso ao léxico"(13), "nomeação automática rápida"(15), "recuperação lexical”(16) e "velocidade de nomeação"(17).

Com o objetivo de comparar as tarefas de nomeação rápida de cores, letras, números, objetos, números/letras e letras/números/cores alternados foi realizada uma pesquisa ${ }^{(18)}$ com 71 crianças distribuídas em três grupos: grupo 1 composto por crianças com distúrbio de leitura; grupo 2, comporto por crianças com déficit de atenção e hiperatividade e grupo 3 (grupo controle), composto por crianças sem distúrbio de leitura. O grupo controle revelou que as crianças do grupo com distúrbios de leitura apresentaram maior lentidão em tarefas de nomeação rápida de letras e números do que as dos grupos controle e as do grupo com déficit de atenção e hiperatividade. Neste estudo foi verificado pelos autores o efeito de idade, pois as crianças mais novas do grupo com distúrbio de leitura apresentaram desempenho inferior em todas as tarefas de nomeação, enquanto as crianças mais velhas do grupo de crianças com distúrbio de leitura apresentaram desempenho inferior apenas em tarefas de nomeação rápida de letras e números. As crianças do grupo com déficit de atenção e hiperatividade apresentaram melhor desempenho em todas as tarefas de nomeação em relação às crianças do grupo com distúrbios de leitura.

Estudos $^{(9)}$ foram realizados com o objetivo de comparar as habilidades fonológicas e os processos de base para aquisição da leitura entre crianças com dislexia, distúrbio específico de linguagem e com desenvolvimento normal. Entre as medidas fonológicas, os autores utilizaram a de nomeação rápida, e verificaram que não ocorreu diferença estatisticamente significante nas provas de nomeação rápida entre o grupo com dislexia e distúrbio específico de linguagem. Entretanto, observou-se diferença significante nas comparações destes dois grupos com o grupo controle. O desempenho do grupo controle foi melhor do que o grupo das crianças com distúrbio específico de linguagem para nomeação rápida de objetos; o grupo controle também obteve melhor desempenho em nomeação de letras do que o grupo de crianças com dislexia.

Foram selecionados 35 estudos realizados no período de 1996 a 2001 para a realização de meta-análise referente à correlação entre nomeação rápida, consciência fonológica e leitura $^{(19)}$. Os autores concluíram que não existem vantagens na obtenção das medidas de consciência fonológica e nomeação rápida em relação às outras medidas preditores de leitura, como a memória; houve correlação entre a nomeação rápida, leitura de pseudo-palavras e soletração, e foi demonstrada a influência da gravidade das dificuldades de leitura para nas correlações entre consciência fonológica e nomeação rápida.

Foi realizado longitudinalmente um estudo ${ }^{(20)}$ com três grupos de crianças, sendo um com baixo risco para dislexia, um com alto risco para dislexia e um com dislexia. Os grupos foram acompanhados desde o jardim até a $2^{\mathrm{a}}$ série. Este estudo teve como objetivo verificar a relação entre as medidas de nível de inteligência, habilidades de leitura (como compreensão, velocidade, decodificação e soletração), consciência fonológica e nomeação rápida. Os autores concluíram que a consciência de fonemas e a nomeação rápida são importantes para a aquisição da leitura no sistema de escrita alfabético, sendo que a consciência fonológica contribuiu significativamente no período pré-escolar - principalmente nas crianças de alto risco para dislexia - enquanto a nomeação rápida contribuiu de forma significativa no período entre a $1^{\mathrm{a}}$ e $2^{\mathrm{a}}$ séries.

Um estudo realizado com crianças leitoras proficientes e crianças com dificuldades de leitura evidenciou que a consciência fonológica, juntamente com a nomeação rápida e leitura de não-palavras, podem ser consideradas importantes 
preditores para o sucesso ou fracasso da leitura ${ }^{(21)}$.

Com objetivo de avaliar a habilidade de nomeação rápida em crianças boas leitoras de $1^{\mathrm{a}}$ e $2^{\mathrm{a}}$ séries do ensino fundamental de escolas públicas e privadas foi realizado um estudo $^{(22)}$ com 62 crianças, de ambos os sexos, divididas em grupo de escola particular (GPA) e grupo de escola pública (GPU). O primeiro grupo foi composto por 27 crianças, sendo seis do sexo feminino e cinco do sexo masculino da $1^{\mathrm{a}}$ série e oito de cada gênero da $2^{\mathrm{a}}$ série. $\mathrm{O}$ segundo grupo foi composto por 35 crianças, sendo nove do sexo feminino e cinco do sexo masculino da $1^{\mathrm{a}}$ série e 10 do sexo feminino e 11 do sexo masculino da $2^{a}$ série. Os instrumentos utilizados para coleta de dados foram as provas de fonologia do ABFW, leitura oral e escrita sob ditado, provas de nomeação rápida de cores, figuras, letras e números. Os resultados evidenciaram diferenças estatisticamente significante no desempenho dos testes de leitura, escrita e nomeação rápida, conforme as séries. As crianças de escola pública tiveram pior desempenho do que as de escola particular, independente das séries. As correlações entre leitura, escrita e nomeação rápida mostraram que quanto maior o tempo de leitura, maior o tempo de nomeação rápida; e quanto maior a acurácia em leitura e escrita, menor é o tempo de nomeação rápida. Os itens alfanuméricos (letras e números) apresentaram relação com a leitura e a escrita, sugerindo que a nomeação rápida esteja associada à velocidade de processamento e ao processamento fonológico, devido às correlações com o tempo e acurácia de leitura.

A percepção de cores em 14 crianças com transtorno do déficit de atenção e hiperatividade (TDAH) foi analisada, e os autores compararam o seu desempenho com o de crianças do grupo controle. Foi utilizado o Teste de Stroop para cores. Os resultados revelaram que as crianças com TDAH apresentaram alteração quanto à discriminação das cores amarelo-azul, vermelho-verde, além de lentidão para nomeação das cores ${ }^{(23)}$.

Com base no exposto acima e considerando que a criança, ao entrar na escola, necessitará integrar informação visual com representações fonológicas estocadas - necessárias para realização da leitura -, este estudo tem como objetivo caracterizar o desempenho de escolares com dislexia e com transtorno do déficit de atenção e hiperatividade em provas de nomeação automática rápida e comparar o desempenho destes escolares com aqueles sem dificuldades de aprendizagem.

\section{MÉTODOS}

Este estudo foi realizado após aprovação do Comitê de Ética em Pesquisa da Universidade Estadual de Campinas/ UNICAMP sob o protocolo número 107/2004.

\section{Sujeitos}

Fizeram parte deste estudo 30 crianças do sexo masculino (100\%), com idade entre 8 anos e 4 meses a 12 anos e 11 meses, sem matriculadas em classes de $2^{\mathrm{a}}$ a $4^{\mathrm{a}}$ séries no ensino público fundamental.
Os sujeitos do GI e GII participantes desta pesquisa fazem parte de um grupo de escolares diagnosticados interdisciplinarmente no Ambulatório de Neuro-Dificuldades de Aprendizagem do Hospital das Clínicas da Universidade Estadual de Campinas - HC/FCM/UNICAMP. Os escolares foram pareados por idade e escolaridade subdivididos em três grupos (Tabela 1):

Grupo I (GI): composto por 10 escolares com diagnóstico interdisciplinar de transtorno do déficit de atenção e hiperatividade (TDAH);

Grupo II (GII): composto por 10 escolares com diagnóstico interdisciplinar de dislexia;

Grupo III (GIII): composto por 10 escolares sem dificuldades de aprendizagem provenientes de escola pública municipal e pareados segundo idade e escolaridade com o GI e GII.

Tabela 1. Distribuição dos escolares segundo média etária e escolaridade

\begin{tabular}{llccc}
\hline Grupos & Média etária & \multicolumn{3}{c}{ Escolaridade } \\
& & $2^{\mathrm{a}}$ & $3^{\mathrm{a}}$ & $4^{\mathrm{a}}$ \\
\hline GI & 9 anos e 6 meses & $30 \%$ & $40 \%$ & $30 \%$ \\
GII & 9 anos e 6 meses & $30 \%$ & $40 \%$ & $30 \%$ \\
GIII & 9 anos e 6 meses & $30 \%$ & $40 \%$ & $30 \%$ \\
\hline
\end{tabular}

Os escolares do GIII, que constituíram o grupo controle deste estudo foram submetidos às mesmas avaliações interdisciplinares (neurológica infantil, fonoaudiológica e neuropsicológica) que o GI e GII no Ambulatório de NeuroDificuldades de Aprendizagem do Hospital das Clínicas da Universidade Estadual de Campinas - HC/FCM/UNICAMP.

\section{Procedimentos}

Os escolares, após assinatura do Termo de Consentimento pelos pais ou responsáveis autorizando o início da pesquisa, foram submetidos à aplicação do Teste de Nomeação Automática Rápida - RAN ${ }^{(6)}$, utilizado para medir a velocidade de nomeação. O procedimento é composto por quatro subtestes para nomeação de cores, dígitos, letras e objetos. Os subtestes são compostos por cinco estímulos diferentes, os quais alternam-se entre si, formando 10 linhas sequienciais em um total de 50 estímulos. O subteste de cores é composto pelas cores verde, vermelho, preto, azul e amarelo. O subteste de letras é composto pelas letras "p", "d", "o", "a" e "s". O subteste de dígitos é composto pelos números "6", "2", “4", "9" e "7". O subteste de objetos é composto por figuras dos seguintes objetos: pente, guarda-chuva, chave, relógio e tesoura.

Os escolares foram orientados a nomear o mais rapidamente possível os estímulos visuais apresentados em cada prancha, seguindo o estímulo para nomeação da esquerda para a direita e de cima para baixo, na menor velocidade possível. O tempo foi registrado com cronômetro.

Cada escolar foi testado individualmente durante uma sessão de aproximadamente 40 minutos. 


\section{Análise estatística}

Nesta etapa foi realizada análise estatística dos resultados, pela aplicação da Análise de Variância, ajustada pelo Teste de Homogeneidade de Variâncias de Levene, com o intuito de verificar possíveis diferenças das médias entre os grupos nas variáveis de interesse.

A análise estatística foi complementada pela aplicação dos Testes de Múltipla Escolha de Tukey e/ou Dunnett com base no Teste de Homogeneidade de Variâncias de Levene, com o objetivo de comparar os pares de grupos. Os resultados estatisticamente significantes serão assinalados por asterisco $(*)$.

Adotamos o nível de significância de $5 \%(0,050)$ para a aplicação dos testes estatísticos, ou seja, quando o valor da significância calculada (p) for menor do que $5 \%(0,050)$ observamos uma diferença "estatisticamente significante".

\section{RESULTADOS}

A Tabela 2 apresenta o desempenho dos grupos na prova de nomeação automática rápida. Com a aplicação da análise variância, foi verificada diferença estatisticamente significante entre os grupos, evidenciando-se melhor desempenho do grupo controle em relação ao grupo de escolares com transtorno do déficit de atenção e do grupo de escolares com dislexia.

Tabela 2. Distribuição da média, desvio-padrão e p-valor do desempenho dos escolares dos GI, GIl e GIII nos subtestes no Teste de Nomeação Automática Rápida (RAN).

\begin{tabular}{llcccc}
\hline Variável & Grupo & $\mathrm{n}$ & Média & $\begin{array}{c}\text { Desvio- } \\
\text { padrão }\end{array}$ & $\begin{array}{c}\text { Significância } \\
(\mathrm{p})\end{array}$ \\
\hline CORES & GI & 10 & 43,08 & 13,32 & $0,008^{*}$ \\
& GII & 10 & 55,38 & 7,53 & \\
& GIII & 10 & 41,04 & 8,62 & \\
& Total & 30 & 46,50 & 11,71 & \\
\hline DIGITOS & GI & 10 & 30,15 & 6,24 & $<0,001^{*}$ \\
& GII & 10 & 47,96 & 17,16 & \\
& GIII & 10 & 26,50 & 3,90 & \\
& Total & 30 & 34,87 & 14,11 & \\
\hline LETRAS & Gl & 10 & 32,51 & 12,96 & $0,006^{*}$ \\
& GII & 10 & 43,05 & 9,70 & \\
& GIII & 10 & 27,63 & 5,78 & \\
& Total & 30 & 34,40 & 11,60 & \\
\hline OBJETOS & Gl & 10 & 57,32 & 11,78 & $<0,001^{*}$ \\
& GII & 10 & 77,58 & 17,05 & \\
& GIII & 10 & 51,57 & 10,06 & \\
& Total & 30 & 62,16 & 17,13 & \\
\hline
\end{tabular}

Para verificar o desempenho dos grupos nos quatro subtestes do RAN utilizamos os testes de múltipla escolha, sendo o Teste de Tukey para comparação do desempenho dos grupos para cores, letras e objetos e o Teste de Dunnett para dígitos. Na Tabela 3 evidenciamos que o grupo de escolares com TDAH obteve desempenho superior em cores quan- do comparado ao grupo de escolares com dislexia e que o grupo de escolares com dislexia teve desempenho inferior aos escolares do grupo controle. Estabeleceu-se, desta forma, diferença estatisticamente significante, o mesmo ocorrendo com os subtestes de dígitos e objetos. Nesta tabela também é possível observar que, para o subteste de letras, os escolares com dislexia apresentaram desempenho inferior aos escolares do grupo controle, o que, assim como no caso anterior, foi evidenciado estatisticamente.

Tabela 3. Distribuição da média, desvio-padrão e p-valor do desempenho dos escolares dos GI, GIl e GIII nos subtestes no Teste de Nomeação Automática Rápida (RAN).

\begin{tabular}{lllll}
\hline Grupos & Cores & Dígitos & Letras & Objetos \\
\hline GI x GII & $p=0,030^{*}$ & $p=0,030^{\star}$ & $p=0,062$ & $p=0,006^{\star}$ \\
GI x GIII & $p=0,895$ & $p=0,345$ & $p=0,522$ & $p=0,603$ \\
GII x GIII & $p=0,010^{\star}$ & $p=0,009^{\star}$ & $p=0,005^{\star}$ & $p<0,001^{\star}$ \\
\hline
\end{tabular}

\section{DISCUSSÃO}

Na realização de atividades como leitura de palavras isoladas ou texto, é necessário um processamento visual refinado dos sinais gráficos para que se dê a realização de varredura textual para identificação das partes constituintes da palavra e, conseqüentemente, sua fixação, codificação e posterior compreensão. Tal habilidade é requisitada, portanto, logo que a criança inicia a alfabetização. Entretanto, devemos considerar que, relacionado a este processamento visual, está o processamento lingüístico da leitura, o qual realiza a identificação da palavra mediante o processo de decodificação fonológica, sendo este auxiliado pelo processamento auditivo. Tal processo permite a conversão dos sinais gráficos em representações fonológicas.

Esta interação entre processamento visual, lingüístico e auditivo exige uma função executiva atuante que, quando comprometida, altera a aquisição de estratégias de leitura, mais especificamente de estratégias fonológicas para leitura, resultando em falhas na automatização da decodificação fonológica. Tais falhas geralmente impedem o acesso ao significado das palavras e textos, comprometendo assim o objetivo final da leitura, ou seja, a compreensão do texto lido.

As crianças que apresentam falhas atencionais ou de processamento da informação terão dificuldade para acionar um processamento visual refinado, o que comprometerá o acesso fonológico exigido para a realização da leitura e escrita de um sistema alfabético ${ }^{(23-24)}$.

Os achados deste estudo evidenciaram que os escolares com TDAH apresentam dificuldades para nomear rapidamente estímulos como cores, dígitos, letras e objetos quando comparados ao grupo controle. Entretanto, os escolares com dislexia apresentam desempenho inferior tanto em relação ao grupo de escolares com TDAH como em relação ao grupo controle.

Estes achados vão de encontro aos resultados encontrados na literatura ${ }^{(18,24)}$, segundo os quais o fato de crianças com TDAH apresentarem melhores desempenhos em nome- 
ação rápida em relação aos disléxicos indica que o processamento lingüístico destas crianças não é tão comprometido como no disléxico. Esta observação pode ser evidenciada em tarefas de leitura e escrita, nas quais as crianças com TDAH têm melhor domínio ortográfico do que crianças com dislexia.

Como a nomeação rápida é parte do processamento fonológico ${ }^{(8-11)}$, é esperado que escolares com quadro de dislexia apresentem desempenho inferior nos subtestes do RAN em relação ao grupo de escolares com TDAH e ao grupo controle, pelo fato da ocorrência de falhas no processamento fonológico, determinadas genética e neurologicamente $^{(25)}$. Outros estudos ${ }^{(11,26)}$ também evidenciaram as dificuldades de crianças disléxicas em subtestes de nomeação automática rápida.

Entretanto, também devemos ressaltar que, no presente estudo, tanto os escolares com TDAH como os escolares com dislexia apresentaram comprometimento em nomeação automática rápida; porém, os escolares do grupo com dislexia apresentaram desempenho inferior ao grupo com TDAH.

A nomeação automática rápida, avaliada pelo teste RAN, é uma habilidade de linguagem que faz parte do processamento fonológico. Diversos autores ${ }^{(10,27)}$ descreveram que o RAN pode oferecer informação referente à atenção, percepção, seqüencialização, memória, acesso ao léxico e processos articulatórios.

Estudos mais recentes ${ }^{(11,19-22,28)}$ apontam para a necessidade de pesquisas que estabeleçam relações entre os subtestes de nomeação automática rápida e habilidades fonológicas e de leitura e escrita.

Neste estudo, o que evidenciamos foi que os escolares com TDAH apresentaram melhor desempenho no subteste de letras, igualando o desempenho ao do grupo de escolares normais, compatível com os estudos que afirmaram que o desempenho lingüístico desta população é superior ao da população de disléxicos ${ }^{(18,23)}$.

Ainda é importante ressaltar que os efeitos de supressão articulatória e de extensão de palavras estão localizados no processo de rechamada subvocal, e que, se houver qualquer alteração no processamento lingüístico da informação, este inibirá o acesso ao estoque fonológico, gerando falhas para nomeação de palavras com maior extensão, como na nomeação de cores e objetos ${ }^{(29)}$.

\section{CONCLUSÃO}

Os achados deste estudo nos possibilitaram concluir que: - Os escolares do grupo com transtorno do déficit de atenção e hiperatividade e do grupo com dislexia apresentam desempenho inferior ao do grupo controle nos subtestes de cores, dígitos, letras e objetos da prova de nomeação automática rápida (RAN);

- Os escolares do grupo com transtorno do déficit de atenção e hiperatividade apresentam melhor desempenho em cores, dígitos, letras e objetos que os escolares do grupo de dislexia.

A nomeação rápida é considerada na literatura internacional como um preditor para o desenvolvimento da leitura e da escrita. O presente estudo demonstrou que os escolares que lêem de acordo com o esperado para idade e escolaridade apresentaram melhor desempenho em relação ao grupo de escolares com transtorno do déficit de atenção e hiperatividade e o grupo com dislexia, demonstrando que tal habilidade pode ser considerada um pré-requisito para o desempenho em leitura. Sendo assim, há necessidade de continuidade de pesquisas que estabeleçam a relação desta habilidade da linguagem com outras habilidades como consciência fonológica, memória de trabalho, leitura e escrita nas populações aqui analisadas para verificação do impacto desta habilidade no processo de aprendizagem da leitura.

\begin{abstract}
Purpose: To characterize the performance of students with attention deficit hyperactivity disorder and dyslexia in rapid automatized naming and to compare it to the performance of children whose reading ability is considered to be in accordance to age and schooling. Methods: A number of 30 students aged 8 to 12 years old attending $2^{\text {nd }}$ to $4^{\text {th }}$ grades in a public school participated in this study, divided into three groups, one formed by students with attention deficit and hyperactivity disorder, another one formed by students with dyslexia, and the other formed by students with good academic performance. Results: The results revealed statistically significant differences, evidencing superior performance of the control group when compared to both the group of students with attention deficit and hyperactivity and the group with dyslexia in the subtests of colors, digit span, letters and objects. The results also revealed superior performance of the group of students with attention deficit compared to the group with dyslexia in rapid automatized naming. Conclusion: Good readers showed better performance when compared to both students with attention deficit and hyperactivity disorder and dyslexia, showing that this ability can be a prerequisite to reading development.
\end{abstract}

KEYWORDS: Attention deficit disorder with hyperactivity; Dyslexia; Underachievement; Reading 


\section{REFERÊNCIAS}

1. Faraone SV. Report from the $4^{\text {th }}$ international meeting of the attention deficit hyperactivity disorder molecular genetics network. Am J Med Genet B Neuropsychiatr Genet. 2003;121(1):55-9.

2. Diagnostic and statistical manual of mental disorders: DSM-IV. $4^{\text {th }}$ ed. Washington: American Psychiatric Association; 2000.

3. Berger M, Yule W, Rutter M. Attainment and adjustment in two geographical areas. II - The prevalence of specific reading retardation. Br J Psychiatry. 1975;126:510-9.

4. Capellini SA. Eficácia do programa de remediação fonológica em escolares com distúrbio de leitura e distúrbio de aprendizagem [tese]. Campinas: Faculdade de Ciências Medicas da Universidade Estadual de Campinas; 2001.

5. Ciasca SM, Capellini SA, Tonelloto JMF. Distúrbios específicos de aprendizagem. In: Ciasca SM, organizadora. Distúrbio de aprendizagem: proposta de avaliação interdisciplinar. São Paulo: Casa do Psicólogo; 2003. p. 55-66.

6. Denckla MB, Rudel R. Rapid "automatized" naming of pictured objects, colors, letters and numbers by normal children. Cortex. 1974;10(2):186202.

7. Vukovic RK, Siegel LS. The double-deficit hypothesis: a comprehensive analysis of the evidence. J Learn Disabil. 2006;39(1):25-47.

8. Wagner RK, Torgesen JK, Rashotte CA, Hecht SA, Barker TA, Burgess $\mathrm{SR}$, et al. Changing relations between phonological processing abilities and word-level reading as children develop from beginning to skilled readers: a 5-year longitudinal study. Dev Psychol. 1997;33(3):468-79.

9. Scheltinga F, Van der Leij A, Van Beinun F. Importance of phonological skills and underlying processes to reading achievement: a study on dyslexic and specific language impaired children. IFA Proc. 2003;(25):21-30.

10. Sprugevica I, Hoien T. Early phonological skills as a predictor of reading acquisition: a follow-up study from kindergarten to the middle of grade 2. Scand J Psychol. 2003;44(2):119-24.

11. Brizzolara D, Chilosi A, Cipriani P, Di Filippo G, Gasperini F, Mazzotti $\mathrm{S}$, et al . Do phonologic and rapid automatized naming deficits differentially affect dyslexic children with and without a history of language delay? A study of Italian dyslexic children. Cogn Behav Neurol. 2006;19(3):141-9.

12. Wolf M, Bowers PG. The double-deficit hypothesis for the developmental dyslexias. J Educ Psychol. 1999;91:415-38.

13. Wagner RK, Torgesen JK. The nature of phonological processing and its causal role in the acquisition of reading skills. Psychol Bull. 1987;101(2):192-212.

14. Wolf M, Bowers PG. Naming-speed processes and developmental reading disabilities: an introduction to the special issue on the doubledeficit hypothesis. J Learn Disabil. 2000;33(4):322-4.

15. Cutting LE, Denckla MB. The relationship of rapid serial naming and word reading in normally developing readers: an exploratory model. Read Writ. 2001;14(7-8):673-705.
16. Allor $\mathrm{JH}$. The relationship of phonemic awareness and rapid naming to reading development. Learn Disab Q. 2002;25(1):47-57.

17. Kirby JR, Parrila RK, Pfeiffer SL. Naming speed and phonological awareness as predictors of reading development. J Educ Psychol. 2003;95(3):453-64.

18. Semrud-Clikeman M, Guy K, Griffin JD, Hynd GW. Rapid naming deficits in children and adolescents with reading disabilities and attention deficit hyperactivity disorder. Brain Lang. 2000;74(1):70-83.

19. Swanson HL, Howard CB, Sáez L. Do different components of working memory underlie different subgroups of reading disabilities? J Learn Disabil. 2006;39(3):252-69.

20. Cardoso-Martins C, Pennington BF. The relationship between phoneme awareness and rapid naming skills and literacy acquisition: the role of development period and reading ability. Sci Stud Read. 2004;8(1):2752.

21. Savage RS, Frederickson N, Goodwin R, Patni U, Smith N, Tuersley L. Relationships among rapid digit naming, phonological processing, motor automaticity, and speech perception in poor, average, and good readers and spellers. J Learn Disabil. 2005;38(1):12-28.

22. Simões VF. Estudo do desempenho de crianças das séries iniciais: do ensino fundamental I em testes de leitura, escrita e nomeação rápida [Tese]. São Paulo: Faculdade de Filosofia, Letras e Ciências Humanas da Universidade de São Paulo; 2006.

23. Banaschewski T, Ruppert S, Tannock R, Albrecht B, Becker A, Uebel $\mathrm{H}$, et al. Colour perception in ADHD. J Child Psychol Psychiatry. 2006;47(6):568-72.

24. Van der Leij A, Morfidi E. Core deficits and variable differences in Dutch poor readers learning English. J Learn Disabil. 2006;39(1):7490.

25. Galaburda AM. Dyslexia: a molecular disorder of neuronal migration. Ann Dyslexia. 2005;55(2):151-65.

26. Nikolopoulos D, Goulandris N, Hulme C, Snowling MJ. The cognitive bases of learning to read and spell in Greek: evidence from a longitudinal study. J Exp Child Psychol. 2006;94(1):1-17.

27. Schatschneider C, Carlson CD, Francis DJ, Foorman BR, Fletcher JM. Relationship of rapid automatized naming and phonological awareness in early reading development: implications for the double-deficit hypothesis. J Learn Disabil. 2002;35(3):245-56.

28. Ferreira TL, Capellini SA, Ciasca SM, Tonelotto JMF. Desempenho de escolares leitores proficientes no teste de nomeação automatizada rápida - RAN. Temas Desenvolv. 2003;12(69):26-32.

29. Gathercole SE, Baddeley AD. Working memory and language. Hove: Lawrence Erlbaum; 1995. 